\title{
Predicting violent reconvictions using the HCR-20
}

Nicola S. Gray, John Taylor and Robert J. Snowden

\section{Background}

Risk assessment of future violent acts is of great importance for both public protection and care planning. Structured clinical assessments offer a method by which accurate assessments could be achieved.

\section{Aims}

To test the efficacy of the Historical, Clinical and Risk Management Scales (HCR-20) structured risk assessment scheme on a large sample of male forensic psychiatric patients discharged from medium secure units in the UK.

\section{Method}

In a pseudo-prospective study, 887 male patients were followed for at least 2 years. The HCR-20 was completed using only pre-discharge information, and violent and other offending behaviour post-discharge was obtained from official records.

\section{Results}

The HCR-20 total score was a good predictor of both violent and other offences following discharge. The historical and risk sub-scales were both able to predict offences, but the clinical sub-scale did not produce significant predictions. The predictive efficacy was highest for short periods (under 1 year) and showed a modest fall in efficacy over longer periods (5 years).

\section{Conclusions}

The results provide a strong evidence base that the HCR-20 is a good predictor of both violent and non-violent offending following release from medium secure units for male forensic psychiatric patients in the UK.

\section{Declaration of interest}

None.
It is government policy that all mental health service users are assessed by specialist mental health services with regard to the potential risk of harm to others. ${ }^{1}$ Many studies have illustrated that unaided clinical judgement as to who will commit future violent acts is poor, ${ }^{2,3}$ and thus instruments have been produced that might aid the clinician in this endeavour. Among the most promising is the Historical, Clinical and Risk Management Scales (HCR-20) violence risk assessment instrument, ${ }^{4}$ an example of a structured clinical judgement scheme. The HCR-20 consists of 20 items that clinicians must consider before making their judgement. The items were chosen because of their alleged connection to violent behaviour, and thus the instrument makes sure that these factors receive due consideration. In justifying the scoring of each item (e.g. 'history of substance misuse'), the scheme identifies the factors and context that contribute to the risks for that individual, and thus aids in the formulation of the risk and management.

The HCR-20 was developed in North America and therefore the vast majority of the evidence base for its efficacy is in that population. In the UK there have only been small-scale studies, both in terms of number of patients assessed and the duration of the prediction interval. ${ }^{5-8}$ In this paper we report on a largescale study of the HCR-20 for assessment of patients being discharged from medium secure units in the UK. As with most research studies of the HCR-20, we have used the 'score' obtained from adding the item scores (i.e. in an actuarial manner).

\section{Method}

The study was a pseudo-prospective case-note analysis of patients discharged from independent sector, medium secure facilities in the UK. The dependent variable was the occurrence of an offence after discharge from the hospital, information obtained from the UK Home Office. Type of offence was also noted and we grouped offences as either 'violent' (all offences classified as violence against the person by the Home Office: kidnap, criminal damage endangering life, robbery, rape and indecent assault) or 'any' (all offences). Time to offence was calculated as the difference between the discharge date and the time of the reconviction for the subsequent offence (the interval between offence and reconviction was often unknown).

\section{Participants}

Patients were discharged from four independent-sector medium secure units run by Partnerships in Care plc (Llanarth Court, Kneesworth House, Stockton Hall and Redford Lodge), between December 1992 and September 2001. The total sample consisted of 996 male patients with a mean age at discharge of 37.7 years (s.d.=9.2, range 16.9-71.2). Most patients (69.2\%) were White, $21.6 \%$ were of Black Caribbean or Black African origin, $2.4 \%$ were of Asian origin, $1.5 \%$ were of other or mixed ethnicity and 5.2\% were of unknown ethnicity. The mean length of stay within the medium secure service was 436 days (s.d. $=510$, range 7-3785).

Primary diagnosis was divided into affective disorder (9.9\%), personality disorder $(9.0 \%)$, schizophrenia or psychotic disorder (56.2\%), drug-induced psychosis (4.7\%), mental retardation $(8.5 \%)$ and 'other' diagnoses (8.4\%: anxiety disorder, developmental disorder, organic disorder and epilepsy), with 3.2\% patients of unknown diagnosis. Diagnoses were made by a consultant psychiatrist upon admission to hospital using ICD10 criteria. $^{9}$

It was not possible to gather exactly the same data for all the participants. Thus, many of the analyses below are on sub-samples of this overall population. For each sub-sample used (e.g. those with a valid HCR-20 score and a follow-up of 5 years) we compared the above patient characteristics (e.g. age, diagnosis) with those of the overall population. No significant difference was found.

\section{Measures}

The HCR-20 consists of 20 items: 10 items related to historical factors (e.g. employment problems, history of mental illness), 5 items related to current clinical presentation (e.g. lack of insight, 
current symptoms of major mental illness) and 5 items related to future risk factors (e.g. lack of personal support, non-compliance with remediation attempts). Each item was scored as 0 (not present), 1 (partially or possibly present) or 2 (present), leading to a maximum total score of 40 , and maximum sub-scale scores of 20 for the historical scale and 10 for the clinical and risk scales. If insufficient information was available we omitted the item score but pro-rated the scale and sub-scales (by taking the average score on scale or sub-scale). If too many items were omitted (more than five in total, two for the historical scale and one for the clinic and risk scales), then the assessment was considered invalid and omitted from the analysis. In all we were able to score 887 patients at their point of discharge.

\section{Procedure}

Ethical approval was obtained from the ethical committee of the School of Psychology, Cardiff University. Four psychologists completed all assessments by access to file-based information. Each assessor was trained on the HCR-20, and on a test sample of 20 cases the raters had a collective interclass correlation of 0.80 . All background psychiatric and mental health reports on the patients were obtained, as were full criminal record history, admission and discharge psychiatric and psychological reports, social work and probation information, and nursing records. Risk assessments were completed masked to outcome following discharge. The data available to us were the date of any reconviction following discharge. Cases of patients reconvicted for a non-violent offence were removed from the analysis of violent offences from the time the non-violent offence occurred, as these individuals might no longer have been at liberty to commit further offences.

\begin{tabular}{|lcc|}
\hline Table 1 Descriptive data for the HCR-20 & \\
\hline HCR-20 scale & Mean (s.d.) & Range \\
\hline Total (0-40) & $18.3(6.2)$ & $0-36$ \\
\hline Historical (0-20) & $11.3(3.7)$ & $0-20$ \\
\hline Clinical (0-10) & $3.2(2.4)$ & $0-10$ \\
\hline Risk (0-10) & $3.7(2.6)$ & $0-10$ \\
\hline & & \\
\hline a. Possible score range in parentheses. & \\
\hline
\end{tabular}

\section{Results}

\section{Risk scores}

Table 1 shows the descriptive statistics for the HCR-20 assessments in this forensic psychiatric sample. The figures resemble those for civil psychiatric patients in North America. ${ }^{10}$ The only notable differences are that the clinical and risk sub-scale scores are a little smaller in our sample.

\section{Prevalence of offending}

Table 2 presents the number of people convicted and not convicted, and the derived base rates, for 'violent' and 'any' convictions. Thus, after 5 years we found that $34 \%$ of our cohort had a new conviction, with $10 \%$ receiving a conviction for a violent offence. Our results (from independent sector hospitals) appear similar to previous data on people discharged from medium secure units in the UK (mainly National Health Service units). Maden et al reported that after 2 years $15 \%$ of those discharged had been convicted, ${ }^{11}$ with $6 \%$ having convictions for a violent offence compared with $19.5 \%$ and $5.4 \%$ respectively in our cohort.

\section{Predictive validity of HCR-20}

We assessed the efficacy of the HCR-20 risk prediction using signal detection theory. The area under the curve (AUC) of the receiver operating characteristic is regarded as a succinct method of quantifying performance of an instrument that is relatively immune to changes in base rate, ${ }^{12}$ and has been used in many previous studies of risk prediction efficacy. In practice, AUCs greater than 0.54 are regarded as small effects, those greater than 0.63 are moderate effects and those greater than 0.71 are large effects. $^{13}$

Table 1 contains the results for predicting violent reconviction and for the prediction of any reconviction. The HCR-20 was a good predictor of violent offences, with AUCs in the 0.70-0.76 range. It is also noticeable that the efficacy of the prediction declines a little with increasing follow-up period and this was a statistically significant trend $(P<0.05)$. The historical sub-scale was also a good predictor (AUC 0.68-0.77) and showed a similar pattern of results to that of the total scale. The risk sub-scale (AUC 0.63-0.69) showed moderate levels of predictive efficacy, with again a trend to decrease with increasing length of follow-up.

\begin{tabular}{|c|c|c|c|c|c|c|c|c|c|c|}
\hline & \multirow{2}{*}{$\begin{array}{c}\text { Convicted/non } \\
\text { convicted } \\
n / N\end{array}$} & \multirow{2}{*}{$\begin{array}{c}\text { Base rate } \\
\%\end{array}$} & \multicolumn{2}{|c|}{ Total } & \multicolumn{2}{|c|}{ History } & \multicolumn{2}{|c|}{ Clinical } & \multicolumn{2}{|c|}{ Risk } \\
\hline & & & AUC & s.e. & AUC & s.e. & AUC & s.e. & AUC & s.e. \\
\hline \multicolumn{11}{|c|}{ Violent conviction } \\
\hline $1 / 2$ year & $14 / 851$ & 1.6 & $0.76 * *$ & 0.05 & $0.77^{* *}$ & 0.06 & 0.61 & 0.05 & $0.69 *$ & 0.07 \\
\hline 1 year & $22 / 810$ & 2.6 & $0.76^{* *}$ & 0.05 & $0.76^{* *}$ & 0.05 & 0.61 & 0.05 & $0.68^{* *}$ & 0.06 \\
\hline 2 year & $43 / 749$ & 5.4 & $0.71 * *$ & 0.04 & $0.71 * *$ & 0.04 & 0.54 & 0.04 & $0.65^{* *}$ & 0.04 \\
\hline 5 year & $55 / 462$ & 10.6 & $0.70^{\star *}$ & 0.04 & $0.68^{* *}$ & 0.04 & 0.57 & 0.04 & $0.63^{* *}$ & 0.04 \\
\hline \multicolumn{11}{|c|}{ Any conviction } \\
\hline $1 / 2$ year & $45 / 842$ & 5.1 & $0.75^{\star \star}$ & 0.03 & $0.75^{\star *}$ & 0.03 & 0.55 & 0.04 & $0.68^{* *}$ & 0.04 \\
\hline 1 year & 98/789 & 11.0 & $0.70 * *$ & 0.03 & $0.69 * *$ & 0.03 & 0.51 & 0.03 & $0.69^{* *}$ & 0.03 \\
\hline 2 year & $173 / 714$ & 19.5 & $0.69^{* *}$ & 0.02 & $0.69 * *$ & 0.02 & 0.51 & 0.02 & $0.67^{* *}$ & 0.02 \\
\hline 5 year & $226 / 434$ & 34.2 & $0.69 * *$ & 0.02 & $0.70 * *$ & 0.02 & 0.52 & 0.02 & $0.66^{* *}$ & 0.02 \\
\hline
\end{tabular}


However, the clinical scale did not produce any statistically significant prediction (AUC 0.54-0.61).

A great difficulty in research into the prediction of violent behaviour is the generally low level of convictions for violent offences (this is not the same as low levels of violent acts - see Discussion). It has been suggested that 'all reconvictions' can be used as a preliminary test-bed for testing violence risk instruments, ${ }^{14}$ as their efficacy appears to be similar for both offence types (implying similar causal factors) but general offending is more prevalent. Table 2 describes these data and supports this notion.

\section{Discussion}

\section{HCR-20 - comparison with previous findings}

Our data clearly show the efficacy of the HCR-20 in this large UK sample of forensic psychiatric patients. In line with previous results, ${ }^{10}$ we find that the total, historical and risk scales are effective in predicting violent convictions, but that the clinical scale has less value in violent risk prediction for this group under these conditions.

There have been few previous studies of the HCR-20 in the UK. Gray et al showed that the HCR-20 was an excellent predictor of in-patient violence over a short period (3 months), with both the historical and clinical scales making significant contributions to the predictions. ${ }^{5}$ Grevatt et al, ${ }^{6}$ again looking at short-term in-patient violence, agreed on the efficacy of the clinical scale of the HCR-20, but did not find any efficacy for the history scale - indeed, inspection of their results seems to suggest less than chance levels. For the prediction of violence outside the institution, Doyle \& Dolan showed that both the total and historical scale of the HCR-20 were good predictors over a short interval of 24 weeks. $^{7}$ They also noted that the addition of dynamic variables (such as the clinical and risk scales of the HCR-20) can improve upon purely historical baseline measures. These results appear in broad agreement with our results for short-term follow-up (see half-year follow-up data), save that we found only modest (and not statistically significant) contributions from the clinical scale. The finding that the clinical scale is of great value for institutional violence, but of less value for longer-term reconvictions, should not be regarded as stating that clinical variables are unimportant in risk prediction. It seems more probable that people admitted to a secure hospital are more likely to be unwell, and that decisions to discharge patients from this setting are only likely to be made when clinical variables are stable and when there is a sensible care plan to manage the mental health of the person. Hence, the lack of predictive value of the clinical scale could be interpreted as the successful management of risk that is caused by clinical variables, rather than the lack of causal contribution to risk from clinical variables (see also below for other possible reasons for the poor prediction of the clinical scale).

\section{Prediction interval}

This is the first study to compare predictions of the HCR-20 across different follow-up periods. We found that the HCR-20 prediction efficacy (as defined by signal detection methods) showed a small decline over longer follow-up periods. This is, perhaps, not surprising for the risk scale where the assessor has to consider the person's future environment. Clearly, for such a dynamic measure, as time progresses this environment is liable to change, thus the original risk assessment will no longer be relevant. A similar argument holds for the clinical scale. Again, because this variable is dynamic it may have little relevance even after 6 months. More surprising was the gradual decline in the efficacy of the historical scale over the longer follow-up periods. The reasons for this are unclear, but such a gradual decline over time has also been noted for other risk assessment schemes. ${ }^{14}$

\section{Prevalence of violence}

We found that the prevalence of violent convictions among our sample was quite low and comparable with that of a previous study of patients discharged from medium secure units in the UK. ${ }^{11}$ However, convictions are only the 'tip of the iceberg' of actual acts of violence. Doyle \& Dolan used official records, selfreport and collateral information to define any act of violence, and found $19 \%$ of their sample had committed some act of violence within 6 months ${ }^{7}$ compared with our finding of under $2 \%$ conviction for violence. Although hard comparisons are difficult owing to differences in the exact definitions of violence, time taken for a violent act to lead to a conviction, etc., it seems most likely that the vast majority of violent acts do not lead to a conviction. Thus, the ability of the HCR-20 to predict these violent convictions is all the more impressive, given this large source of 'noise' in the dependent measure.

\section{Concluding remarks}

Our data provide an evidence base for the use of the HCR-20 structured risk assessment scheme for the prediction of violence in male psychiatric patients discharged from secure units in the UK. Further, this study used the HCR-20 in an 'actuarial' manner (i.e. we derived a score by adding together the item scores), whereas the real strength of the HCR-20 lies in its use to guide clinical judgement about risk and therefore about risk management. We note that there is some evidence that structured risk assessments are even more effective when used in this clinical manner. ${ }^{15,16}$

\section{Nicola S. Gray, School of Psychology, Cardiff University, and South Wales Forensic Psychiatric Service, Mid Glamorgan; John Taylor, Partnerships in Care, Borehamwood; Robert J. Snowden, School of Psychology, Cardiff University,} Cardiff, UK

Correspondence: Nicola S. Gray, School of Psychology, Cardiff University, Cardiff CF10 3AT, UK. Email: grayns@cardiff.ac.uk

First received 13 Aug 2007, final revision 16 Nov 2007, accepted 29 Jan 2008

\section{Acknowledgements}

This work was funded by a grant from Partnerships in Care plc. We thank the UK Home office for providing us with information concerning criminal convictions in our cohort of mentally disordered offenders. We are also grateful to Professor Malcolm MacCulloch for help in commencing this project, and to Suzanne Fitzgerald, Sophie Macculloch, Helen Phillips, Caroline Durrant and Sian Chapple for data collection.

\section{References}

1 Department of Health. Effective Care Co-ordination in Mental Health Services: Modernising the Care Programme Approach. HMSO, 2000.

2 Monahan J, Steadman HJ, Silver E, Appelbaum P, Robbins P, Mulvey E, Roth L, Grisso T, Banks S. Rethinking Risk Assessment: The MacArthur Study of Mental Disorder and Violence. Oxford University Press, 2001.

3 Gardner W, Lidz CW, Mulvery EP, Shaw EC. Clinical versus actuarial predictions of violence in patients with mental illness. J Consult Clin Psychol 1996; 64: 602-9.

4 Webster CD, Douglas KS, Eaves D, Hart SD. HCR-20: Assessing Risk for Violence (Version 2). Simon Fraser University, 1997.

5 Gray NS, Hill C, McGleish A, Timmons D, MacCulloch MJ, Snowden RJ. Prediction of violence and self-harm in mentally disordered offenders: a 
prospective study of the efficacy of HCR-20, PCL-R and psychiatric symptomology. J Consult Clin Psychol 2003; 71: 443-51.

6 Grevatt M, Thomas-Peter B, Hughes G. Violence, mental disorder and risk assessment: can structured clinical assessments predict the short-term risk of inpatient violence? J Forens Psychiatr Psychol 2004; 15: 278-92.

7 Doyle $M$, Dolan $M$. Predicting community violence from patients discharged from mental health services. Br J Psychiatry 2006; 189: 520-6.

8 Gray NS, Snowden RJ, MacCulloch S, Phillips H, Taylor J, Macculloch MJ. Relative efficacy of criminological, clinical and personality measures of future risk of offending in mentally disordered offenders: a comparative study of HCR-20, PCL:SV and OGRS. J Consult Clin Psychol 2004; 72: 523-30.

9 World Health Organization. The ICD-10 Classification of Mental and Behavioural Disorders. WHO, 1992.

10 Douglas KS, Ogloff JRP, Nicholls TL, Grant I. Assessing risk for violence among psychiatric patients: the HCR-20 violence risk assessment scheme and the psychopathy checklist: screening version. J Consult Clin Psychol 1999; 67: 917-30
11 Maden A, Scott, F, Burnett R, Lewis GH, Skapinakis P. Offending in psychiatric patients after discharge from medium secure units: prospective national cohort study. BMJ 2004; 328: 1534.

12 Green DM, Swets JA. Signal Detection Theory and Psychophysics. Wiley, 1966.

13 Rice ME, Harris GT. Comparing effect sizes in follow-up studies: ROC area Cohen's $d$ and r. Law Hum Behav 2005; 29: 615-20.

14 Snowden RJ, Gray NS, Taylor J, MacCulloch MJ. Actuarial prediction of violent recidivism in mentally disordered offenders. Psychol Med 2007; 37: 1539-49.

15 Douglas KS, Yoemans M, Boer DP. Comparative validity analysis of multiple measures of violence risk in a sample of criminal offenders. Crim Justice Behav 2005; 32: 479-510.

16 De Vogel V, de Ruiter C. Differences between clinicians and researchers in assessing risk of violence in forensic psychiatric patients. J Forens Psychiatr Psychol 2004; 15: 145-64.

\section{Why psychiatry still needs psychoanalysis}

\section{Jeremy Holmes}

Because illness-bearers, our patients, come to us not just with diagnoses but with their dreams and character. In dreams lie cherished fears and desires. Character is the precipitate of our material and social being - genes, developmental experiences, choices, relationship patterns, strengths and weaknesses. We are largely unaware of these deep structures that constitute the self. Psychoanalysis provides a language for describing the development of character and for reading dreams. The psychiatrist versed in that language can converse with their patients about how they came to be who they are, and how best to cope with the adversities of mental illness. 